EPJ Web of Conferences 70, 00080 (2014)

DOI: $10.1051 /$ epjconf/ 20147000080

(C) Owned by the authors, published by EDP Sciences, 2014

\title{
Recent results from PHENIX on the evolution of hot QCD
}

\author{
Norbert Novitzky for the PHENIX collaboration ${ }^{12 a}$ \\ ${ }^{1}$ University of Jyväskylä \\ ${ }^{2}$ Helsinki Institute of Physics
}

\begin{abstract}
The Relativistic Heavy Ion Collider's (RHIC) energy scan program explores the phase transition between the hadron gas and the quark gluon plasma phases by varying center-of-mass energies from $\sqrt{s_{N N}}=7.7$ to $200 \mathrm{GeV}$ and using various system sizes. The nuclear modification factor of $\pi^{0}$ and the azimuthal anisotropy of charged hadrons were measured in $\mathrm{Au}+\mathrm{Au}$ collisions at c.m. energies $\sqrt{s_{N N}}=39,62.4$ and $200 \mathrm{GeV}$. In addition, we present recent results of direct photon yield and anisotropy measurements in $\mathrm{Au}+\mathrm{Au}$ collisions at c.m. energy $\sqrt{s_{N N}}=200 \mathrm{GeV}$.
\end{abstract}

\section{Introduction}

The physics of heavy ion collisions addresses the question how the nuclear matter behaves at extremely high temperatures and densities $[1,2]$. The Lattice Quantum Chromodynamics predicts a new state of matter at temperatures $T \geq T_{C} \sim 170 \mathrm{MeV}$ [3]. The strongly interacting matter is called Quark Gluon Plasma (QGP) [4], where quarks and gluons can propagate freely over the distances given by a nucleus size. The created fireball expands in every direction and then cools down until the freeze-out stage is reached. In this stage the final state hadrons are formed.

Among the first processes that take place in heavy ion collision there is the hard scattering of the individual partons. Hard scattering processes are characterized by a large momentum transfer and are calculable by methods of the perturbative Quantum Chromodynamics (pQCD) [5]. In the vacuum (in $\mathrm{p}+\mathrm{p}$ collisions) the outgoing partons lose energy during the fragmentation process. In the heavy ion collision environment the medium induces additional radiation what leads to the suppression of high$p_{T}$ particles, the phenomenon known as the "jet quenching" [6]. This phenomenon has been studied at various c.m. energies and the details are given in Section 2.

The low- $p_{T}$ region of the spectra in collision is driven by collective phenomena, where the tools of hydrodynamics are applicable [7, 8]. The initial spatial energy density distribution in the collision zone results in different pressure gradients in different directions with respect to impact parameter plane, defined by the beam axis and the vector connecting the two nuclei centers. The pressure gradient transforms the initial spatial anisotropy into a momentum anisotropy of the final state hadrons. The results of the azimuthal anisotropy measured in wide range of c.m. energies are shown in Section 3.

Another important probe of the medium is the direct photon production. Due to their small electromagnetic coupling (compared to QCD) they leave the medium without further interaction. The

\footnotetext{
a e-mail: Norbert.Novitzky@cern.ch
} 
deconfined medium degrees of freedom are carried by gluons and quarks, thus the $q_{t} \bar{q}_{t} \rightarrow g_{t} \gamma_{t}$ (where $t$ refers to thermal) channel is enhanced. The low- $p_{T}$ photons $\left(p_{T}<3 \mathrm{GeV} / c\right)$ retain the memory about the space-time averaged temperature of the plasma. On the other hand, the high- $p_{T}$ photons are, to the large extent, insensitive to the QGP evolution. Since the production cross section is calculable in $\mathrm{pQCD}$ frame and they are not modified by the medium, the measurement in this $p_{T}$ region in heavy ion provides a valuable information about the initial yield from hard scattering. The direct photon results are summarized in Section 4.

\section{Nuclear Modification of the Inclusive Distribution of Particles}

When a dense, colored medium is formed in a collision of nuclei A+A, outgoing hard scattered parton propagating through the medium loses energy in the radiation process. Therefore, the observed particle yield in $\mathrm{A}+\mathrm{A}$ collision at a given (high) $p_{T}$ is lower than the one expected in scaled up (to corresponding nucleus-nucleus collision) $\mathrm{p}+\mathrm{p}$ reference. This suppression in the inclusive hadron $p_{T}$ spectrum in heavy ion collisions with respect to $\mathrm{p}+\mathrm{p}$ collisions is characterized in terms of the nuclear modification factor

$$
R_{\mathrm{AA}}=\frac{\left(1 / N_{\mathrm{AA}}^{\mathrm{evt}}\right) d^{2} N_{\mathrm{AA}}^{h} / d p_{T} d y}{\left\langle T_{\mathrm{AA}}\right\rangle d^{2} \sigma_{p p}^{h} / d p_{T} d y}
$$

where $\sigma_{p p}^{h}$ is the hadron production cross section in $\mathrm{p}+\mathrm{p}$ collision measured at same c.m. energy, and $N_{\mathrm{AA}}^{\text {evt }}$ is the number of events in heavy ion collisions. The nuclear overlap function is $\left\langle T_{\mathrm{AA}}\right\rangle=\left\langle N_{\text {coll }}\right\rangle / \sigma_{p p}^{\text {inel }}$, where $\left\langle N_{\text {coll }}\right\rangle$ is the number of binary collisions averaged over a range of impact parameters corresponding to the given centrality class estimated from the Glauber model based Monte Carlo [9].
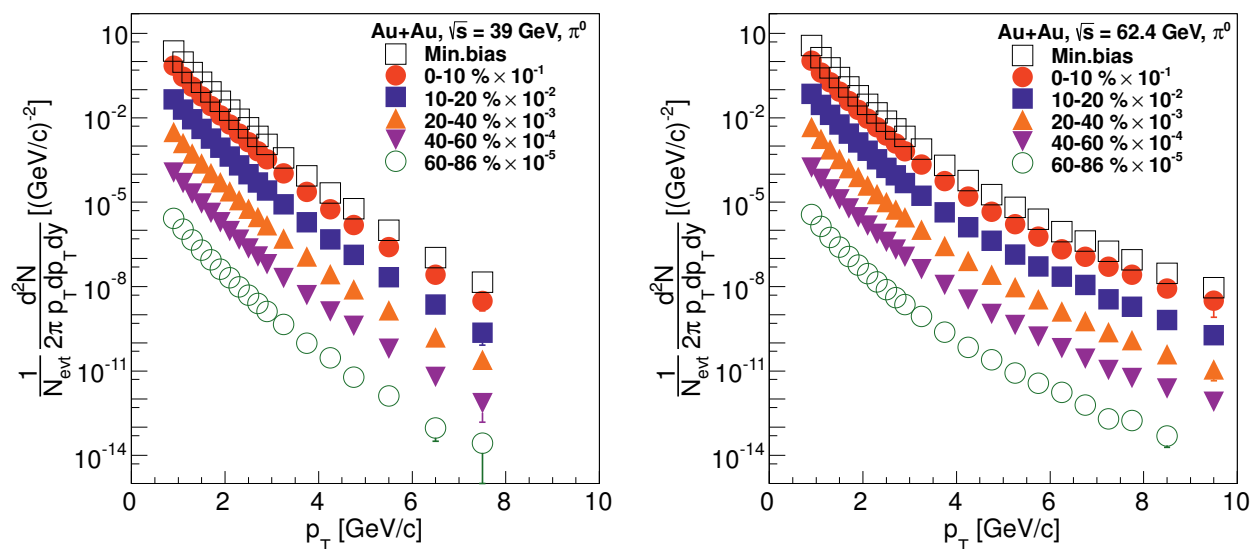

Figure 1. Invariant yield of $\pi^{0}$ measured in $|\eta|<0.35$ in Au+Au collisions at $\sqrt{s_{N N}}=39 \mathrm{GeV}$ (left) and $\sqrt{s_{N N}}=$ $62.4 \mathrm{GeV}$ (right) in minimum bias with empty squares and all centrality classes (indicated in the legend).

The suppression of high- $p_{T}$ hadrons was first measured in $\mathrm{Au}+\mathrm{Au}$ collisions at $\sqrt{s_{N N}}=130$ $\mathrm{GeV}[1,1]$ and later confirmed at $\sqrt{s_{N N}}=200 \mathrm{GeV}$ [1]. The suppression was not observed in $\mathrm{d}+\mathrm{Au}$ collisions at $\sqrt{s_{N N}}=200 \mathrm{GeV}$ [1] which provides a convincing evidence that the suppression is mostly of the final state origin. In 2005, the PHENIX experiment has measured $\pi^{0}$ yields in $\mathrm{Cu}+\mathrm{Cu}$ collisions at three different c.m. energies: $\sqrt{s_{N N}}=22.4,62.4$ and $200 \mathrm{GeV}$ [1]. The $R_{\mathrm{AA}}$ of $\pi^{0}$ 
in the most central collision (0-10\%) shows strong suppression at c.m. energies $\sqrt{s_{N N}}=200$ and $62.4 \mathrm{GeV}$ and no pronounced suppression at $\sqrt{s_{N N}}=22.4 \mathrm{GeV}$. The corresponding $p_{T}$ averaged $\left(2.5<p_{T}<3.5 \mathrm{GeV} / c\right) R_{\mathrm{AA}}$ exhibits a strong centrality dependence in case of $\sqrt{s_{N N}}=62.4$ and 200 $\mathrm{GeV}$ and no centrality dependence at $\sqrt{s_{N N}}=22.4 \mathrm{GeV}$. The low energy scan program was continued in 2010 by collecting $\mathrm{Au}+\mathrm{Au}$ data at $\sqrt{s_{N N}}=39$ and $62.4 \mathrm{GeV}$. The $\pi^{0}$ spectra were measured up to $p_{T}<8$ and $10 \mathrm{GeV} / c$, respectively (see Fig. 1) [1].

For the evaluation of the $R_{\mathrm{AA}}$ a corresponding $\mathrm{p}+\mathrm{p}$ reference at the same collision energy is needed. The $\pi^{0}$ spectrum from $\mathrm{p}+\mathrm{p}$ collisions at $\sqrt{s}=62.4 \mathrm{GeV}$ was measured by the PHENIX experiment up to $p_{T}<7 \mathrm{GeV} / c$. However, the $\mathrm{Au}+\mathrm{Au}$ data extend up to $p_{T}<10 \mathrm{GeV} / c$ (see Fig. 1). To extrapolate the corresponding $\pi^{0}$ spectrum up to $p_{T}<10 \mathrm{GeV} / c$ we used a power law fit of the existing data starting from $p_{T}>4.5 \mathrm{GeV} / c$. The systematic uncertainty resulting from the extrapolation procedure was estimated from a series of fits, where each time one or more randomly selected points were omitted and the remaining points were refitted.

The $\mathrm{p}+\mathrm{p}$ reference at $\sqrt{s}=39 \mathrm{GeV}$ has not been measured by the PHENIX experiment yet and therefore, we used an external measurement from the E706 fix target experiment at Fermilab [1]. Since the E706 experiment has different pseudo-rapidity coverage $(-1<\eta<0.5)$, the original $\pi^{0}$ spectrum was corrected (to $|\eta|<0.35$ ) using PYTHIA8 simulation [1]. An estimate of the systematic uncertainties related to the acceptance correction was deduced from the comparison of the measured (E706) $d N_{-1<\eta<0.5}^{\pi^{0}} / d y$ distributions with the PYHTIA8 simulation.
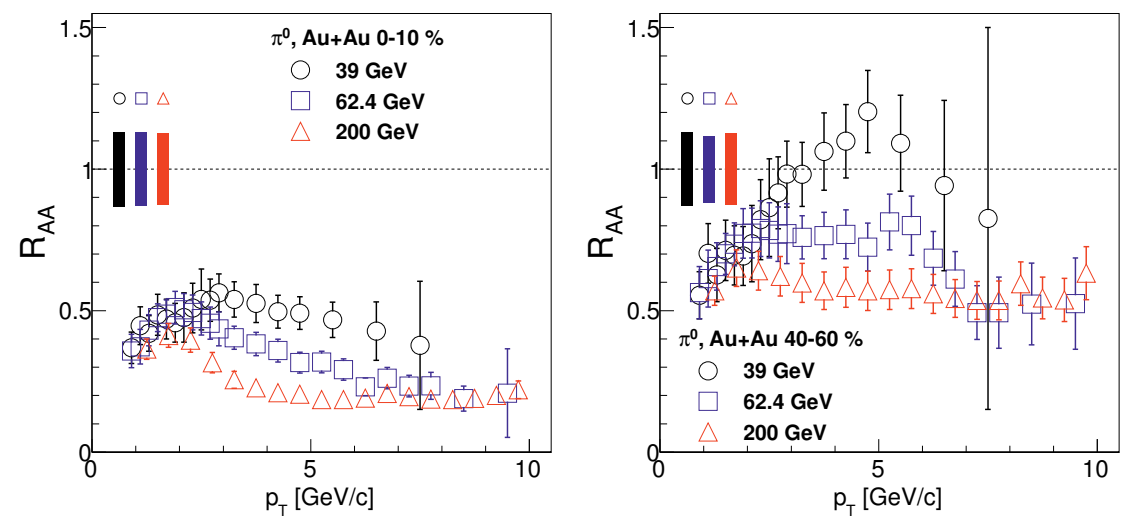

Figure 2. The nuclear modification factor measured in $\mathrm{Au}+\mathrm{Au}$ collisions at $\sqrt{s_{N N}}=39,62.4$ and $200 \mathrm{GeV}$. The error bars give the quadratic sum of the statistical uncertainties and the $p_{T}$ dependent systematic uncertainties. The boxes around unity combine the $\left\langle N_{\text {coll }}\right\rangle$ and the absolute normalization uncertainties. Left panel shows the most central collisions (0-10\%), right panel shows the mid-peripheral collisions (40-60\%).

The $\pi^{0} R_{\mathrm{AA}}$ obtained in $\mathrm{Au}+\mathrm{Au}$ collisions at $\sqrt{s_{N N}}=39,62.4 \mathrm{GeV}$ and $200 \mathrm{GeV}$ are shown in Fig. 2 [1]. The left panel presents the most central $(0-10 \%)$ collisions where the $\pi^{0}$ production at all three energies is suppressed. In the mid-peripheral (40-60\%) collisions the data exhibit substantial suppression at $\sqrt{s_{N N}}=62.4$ and $200 \mathrm{GeV}$ for $p_{T}>4 \mathrm{GeV} / c$, while the data at $\sqrt{s_{N N}}=39 \mathrm{GeV}$ are consistent with unity for $p_{T}>4 \mathrm{GeV} / c$. For both centralities, the $\sqrt{s_{N N}}=62.4$ and $200 \mathrm{GeV}$ data points are comparable for $p_{T}>6 \mathrm{GeV} / c$.

The $p_{T}$ averaged $R_{\mathrm{AA}}$ in range $6<p_{T}<10 \mathrm{GeV} / c$ as a function of the number of participants ( $N_{\text {part }}$, another measure of centrality) is shown in Fig. 3. We observe that the suppression of $\pi^{0}$ at 


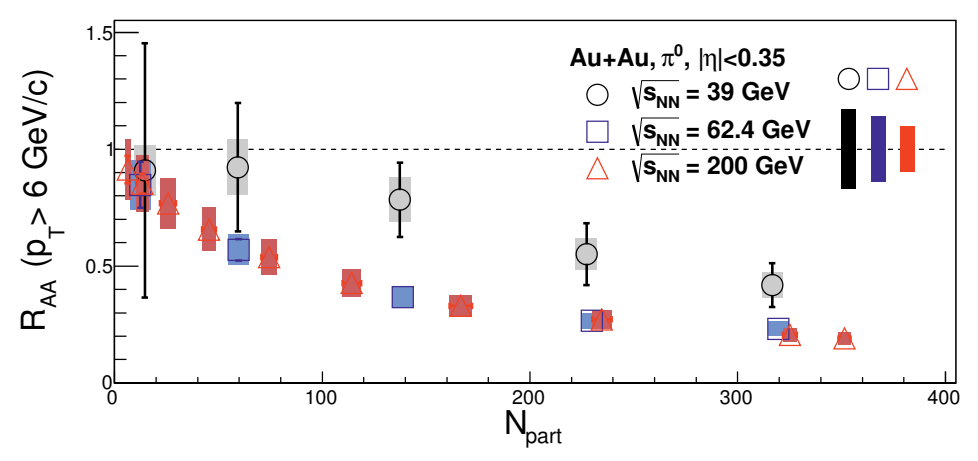

Figure 3. The $\pi^{0}$ nuclear modification factor in $\mathrm{Au}+\mathrm{Au}$ averaged in the interval $6<p_{T}<10 \mathrm{GeV} / c$ as a function of $N_{\text {part }}$ at $\sqrt{s_{N N}}=39,62.4$ and $200 \mathrm{GeV}$. The error bars are calculated as the quadratic sum of the statistical uncertainties and centrality dependent systematic errors and the $\left\langle N_{\text {coll }}\right\rangle$ uncertainties. The boxes around unity are obtained as the systematic uncertainties of the $p+p$ reference and centrality independent systematic uncertainty of $\mathrm{Au}+\mathrm{Au}$ collisions.

$\sqrt{s_{N N}}=39 \mathrm{GeV}$ only starts to dominate in the region $N_{\text {part }}>100$, while it is pronounced already at lower centrality classes at $\sqrt{s_{N N}}=62.4$ and $200 \mathrm{GeV}$.

In summary, the transition from enhancement $\left(R_{\mathrm{AA}}>1\right)$ to suppression $\left(R_{\mathrm{AA}}<1\right)$ was measured around $N_{\text {part }} \sim 100$ in $\mathrm{Au}+\mathrm{Au}$ collisions at $\sqrt{s_{N N}}=39 \mathrm{GeV}$. The measured data suggest, the opacity of the medium becomes less pronounced to other nuclear effects. The data will help to constrain the parameters, such as transport coefficient in the medium, of the energy loss models.

\section{Azimuthal Anisotropy of the Particle Emission}

In the low transverse momentum region $\left(p_{T}<3 \mathrm{GeV} / c\right)$ the hadron dynamics is driven mainly by the collective hydrodynamical phenomena $[7,8]$. The many-body system, such as the QGP, may be described by hydrodynamics when the mean free path of the constituents are much smaller than the size of the system. The initial spatial energy density distribution of the collision zone causes a different pressure gradient in different directions of the produced medium. Due to the finite size of the heavy ions the initial collision zone has an anisotropic (almond) shape for nonzero impact parameters. The pressure gradient consequently results in larger particle production in the reaction plane direction (inplane) as compared to the perpendicular direction (out-plane). The final state momentum anisotropy of the particle production is, traditionally, characterized in terms of Fourier coefficients,

$$
\frac{d^{3} N}{d \phi d p_{T} d y}=\frac{1}{2 \pi} \frac{d^{2} N}{d p_{T} d y}\left(1+\sum_{n} 2 v_{n}\left(p_{T}\right) \cos \left(n\left[\phi-\psi_{n}\right]\right)\right),
$$

where $\psi_{n}$ is the participant event plane and the $v_{n}=\left\langle\cos \left(n\left[\phi-\psi_{n}\right]\right)\right\rangle$ is the $n$-th Fourier coefficient. Flow can address many interesting questions related to the collective phenomena in the medium. In particular, the 2nd harmonic flow coefficient $\left(v_{2}\right)$ at low- $p_{T}$ in Au+Au collisions at $\sqrt{s_{N N}}=200 \mathrm{GeV}$ [1] has revealed that the medium undergoes rapid thermalization and behaves as strongly interacting fluid. The odd terms $(n \geq 3)$ in the Fourier expansion originate from fluctuations in the initial geometry of the collisions.

The determination the charged hadrons $v_{n}$ coefficients was done via long-range two particle correlation. The method is based on the pair-wise distribution in the azimuthal angle difference 


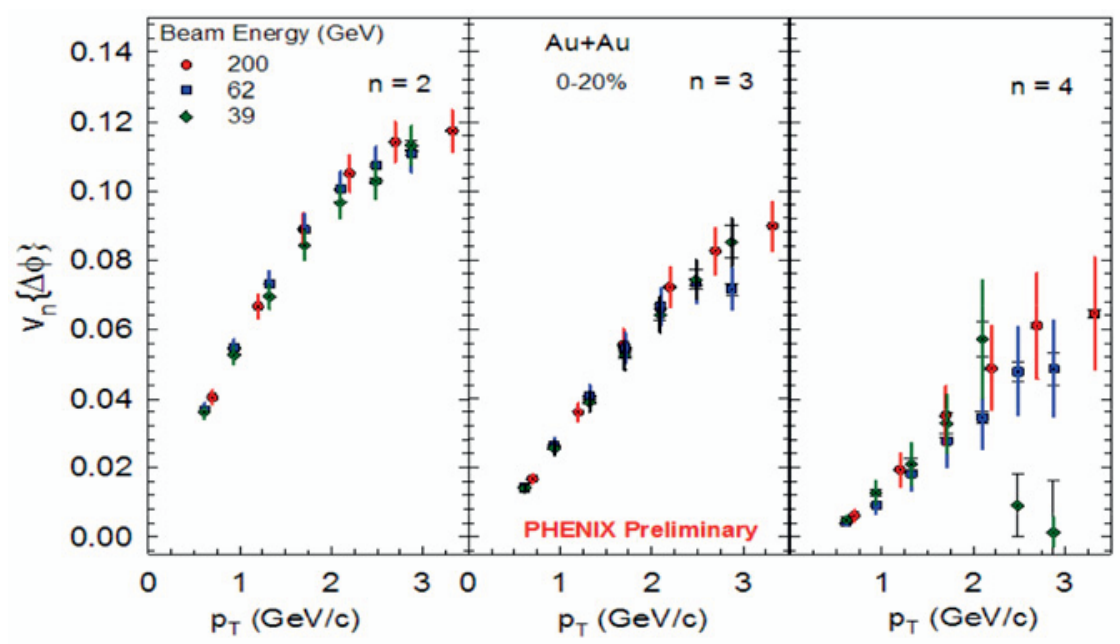

Figure 4. Charged particle $v_{n}\left(p_{T}\right)$ in Au+Au collisions in the centrality class of $0-20 \%$ for $\sqrt{s_{N N}}=200,62.4$ and $39 \mathrm{GeV}$. The error bars give combined statistical and systematic uncertainties. The measurement was done using two particle correlation method between mid- and forward rapidities.

$\left(\Delta \phi=\phi_{1}-\phi_{2}\right)$ between particles across a relatively large pseudorapidity $(\eta)$ gap. One particle is required the mid-rapidity $(\eta \sim 0)$ and the other one is in very forward region. The forward particles were detected by following detectors: the combination of the inner and outer ring of the reaction plane detector (RXNin, $1.0<|\eta|<1.5$, and RXNout, $1.5<|\eta|<2.8$ ), the beam-beam counter (BBC,

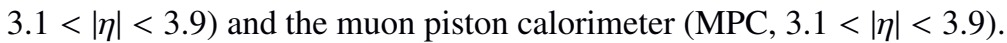

Fig 4. compares the $v_{n}\left(p_{T}\right)$ for $n=2,3$ and 4 of charged particles at three different c.m. energies in $\mathrm{Au}+\mathrm{Au}$ collisions, namely $\sqrt{s_{N N}}=200,62.4$ and $39 \mathrm{GeV}$. The event plane was defined from long range two particle correlation where the forward particle is in the RXNin or RXNout for $\sqrt{s_{N N}}=39$ and $62.4 \mathrm{GeV}$ and in RXNin or MPC for $\sqrt{s_{N N}}=200 \mathrm{GeV}$. Although the collision energy varies by a factor of five, the response of the medium is virtually unchanged.

The initial geometry of the collision, and as a consequence, the initial energy density distribution, changes as a function of the impact parameter, i.e. centrality. The centrality dependence of $v_{n}$ for the two $p_{T}$ ranges is shown in Fig 5. for c. m. energies $\sqrt{s_{N N}}=200,62.4$ and $39 \mathrm{GeV}$. The $N_{\text {part }}$ dependence of the flow at three different c.m. energies does not exhibit any significant difference.

Measurement of the azimuthal anisotropy of produced charged hadrons from the low energy scan do not show any significant deviation down to $\sqrt{s_{N N}}=39 \mathrm{GeV}$. The data suggest the medium created at lower energies is very similar to that created at the top RHIC energy $\sqrt{s_{N N}}=200 \mathrm{GeV}$. The $v_{n}$ results supports the early formation time $\left(0.15<\tau_{0}<0.6 \mathrm{fm} / c\right)$ and similar equation of state (EOS) even at lower c.m. energies.

\section{Direct Photon Results}

Direct photons are emitted in various processes during the entire history of a heavy ion collisions. In first part of the section we focus on the high- $p_{T}$ photons $\left(p_{T}>5 \mathrm{GeV} / c\right)$ which are created mainly from initial hard-scattering processes, such as $q g \rightarrow q \gamma$ ("inverse QCD Compton effect") [1]. The production of hard scattering photons can be affected by nuclear modification (such as shadowing). In 


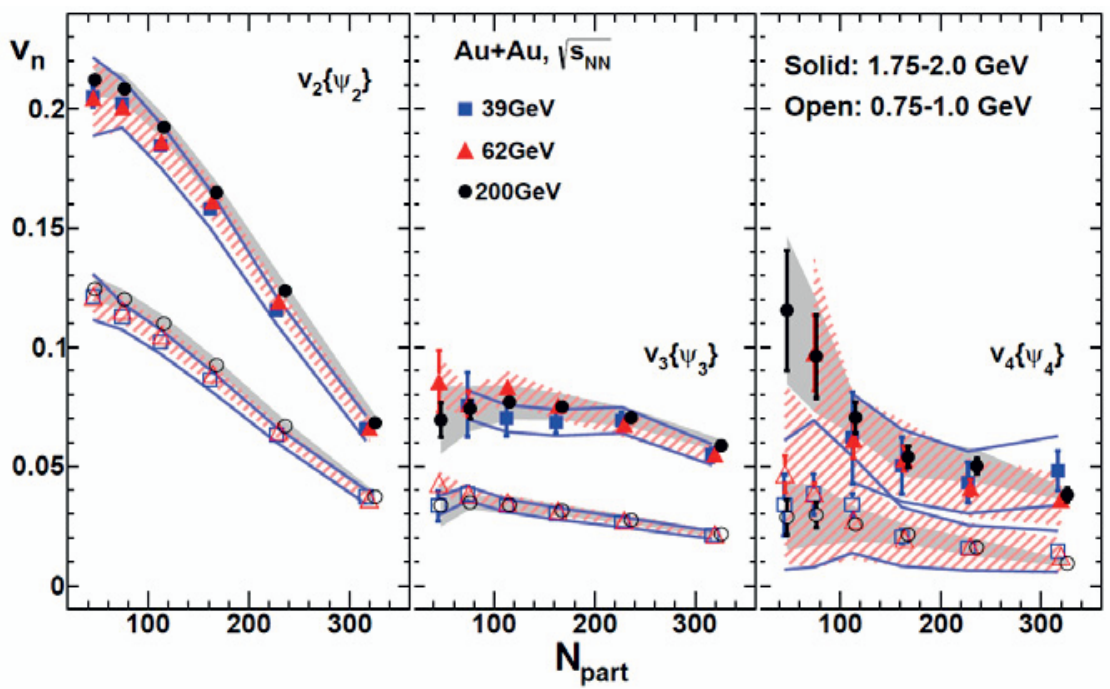

Figure 5. Charged particle $v_{n}$ in $\mathrm{Au}+\mathrm{Au}$ collisions for $1.75<p_{T}<2.0 \mathrm{GeV} / c$ (solid symbols) and $0.75<p_{T}<$ $1.0 \mathrm{GeV} / c$ (open symbols) for $\sqrt{s_{N N}}=200,62.4$ and $39 \mathrm{GeV}$. The error bars give the statistical uncertainties, the bands represent the systematic uncertainties. The measurement was done using two particle correlation method between mid- and forward rapidities.

addition, direct photons at high- $p_{T}$ are created also during processes such as fragmentation of quarks and gluons jets and jet-medium interaction. While direct photon production from the initial hard scattering is isotropic $\left(v_{2} \sim 0\right)$, the fragmentation photon could be radiated with finite anisotropy due to different energy loss of the mother parton along different path length in different directions w.r.t. reaction plane.

The high- $p_{T}\left(p_{T}>4 \mathrm{GeV} / c\right)$ photons are identified using $\pi^{0}$ tagging $(\mathrm{p}+\mathrm{p})$ or statistical subtraction method [2]. The subtraction method is based on a comparison of inclusive photon spectra to the expected background from hadronic decays (mainly $\pi^{0} \rightarrow 2 \gamma$ and $\eta \rightarrow 2 \gamma$ ). Decay photons from hadronic decays were simulated using the kinematics and detector response and subtracted from the inclusive photon yields.

The high- $p_{T}$ direct photon production was measured in $\mathrm{Au}+\mathrm{Au}$ and $\mathrm{p}+\mathrm{p}$ collisions at $\sqrt{s_{N N}}=200$ $\mathrm{GeV}$. The production of direct photons in $\mathrm{p}+\mathrm{p}$ collision is described by the next-to-leading $\mathrm{pQCD}$ as can be seen in Fig 6 left. The photon yield in minimum bias and two different centrality bins (most central, $0-10 \%$, and most peripheral, 60-92\%) of $\mathrm{Au}+\mathrm{Au}$ collisions is compared to $T_{\mathrm{AA}}$ scaled up (see Eq. 1) $p+p$ cross section in Fig 6 right [2]. When comparing to $p+p$ collisions, the direct photon production can be affected by the isospin effect, because the neutron content of the gold nuclei. The isospin effect is expected to be at most $10 \%$ at $p_{T} \sim 20 \mathrm{GeV} / c$. The $R_{\mathrm{AA}}$ values are comparable with the unity in each centrality class and for all $p_{T}>4 \mathrm{GeV} / c$. The azimuthal anisotropy of high$p_{T}$ direct photons $\left(5<p_{T}<13 \mathrm{GeV} / c\right)$ is consistent with zero within uncertainties, see Fig 7 . The production of high- $p_{T}$ photons is consistent with the scenario where they are emitted from initial hardscattering, and then traverse the medium without further interaction. It also implies that the fraction of fragmentation photons is small.

In second part of the section we discuss the low- $p_{T}$ region $\left(p_{T}<3 \mathrm{GeV} / c\right)$ where additional photons may be emitted from the thermalized medium of the partons or hadrons. The additional 

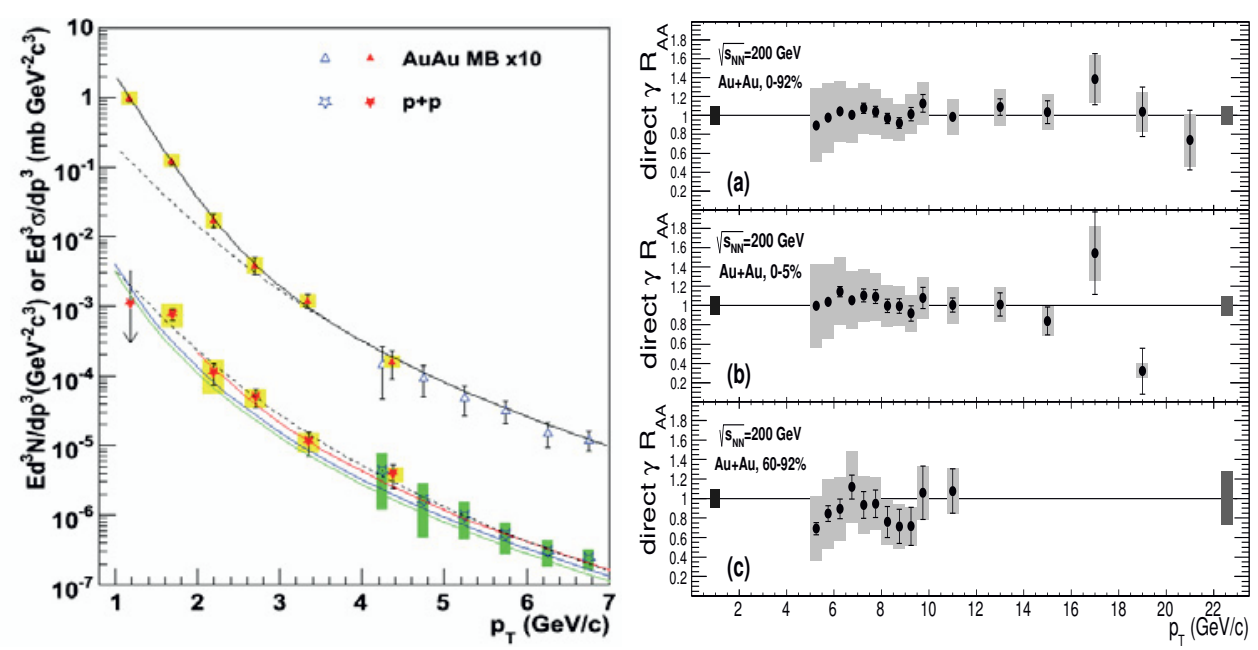

Figure 6. Left: Direct photon invariant cross section in $\mathrm{p}+\mathrm{p}$ collision and the invariant yield in $\mathrm{Au}+\mathrm{Au}$ minimum bias collision at $\sqrt{s_{N N}}=200 \mathrm{GeV}$. Filled points are from the dilepton analyses and the open points are from the calorimeter analysis. The three curves on the $\mathrm{p}+\mathrm{p}$ data show the perturbative QCD calculations [1] and the dashed curve over $\mathrm{Au}+\mathrm{Au}$ data is the power-law fit to the $\mathrm{p}+\mathrm{p}$ data, scaled by $T_{\mathrm{AA}}$ from the Glauber Monte Carlo [9]. The solid curve going through the $\mathrm{Au}+\mathrm{Au}$ data is the exponential plus the $T_{\mathrm{AA}}$ scaled $\mathrm{up} \mathrm{p}+\mathrm{p}$ fit. Right: Direct photon nuclear modification factor $\left(R_{\mathrm{AA}}\right)$ for three different centralities (indicated in legends) in $\mathrm{Au}+\mathrm{Au}$ collisions at $\sqrt{s_{N N}}=200 \mathrm{GeV}$. The error bars represent the statistical uncertainties and the bands show the systematic uncertainties. The boxes around the unity represent the $\left\langle N_{\text {coll }}\right\rangle$ uncertainties.

thermal photons are emitted in the form of real $(\gamma)$ or virtual $\left(\gamma^{*}\right)$ photons from initial QGP phase (e.g. $q \bar{q} \rightarrow g \gamma^{*} \rightarrow g e^{+} e^{-}$and $q g \rightarrow q \gamma$ ) and hadron gas phase (e.g. $\pi \rho \rightarrow \pi \gamma$ and $\pi \pi \rightarrow \rho^{*} \rightarrow \gamma^{*} \rightarrow e^{+} e^{-}$). Hence, they retain the memory on the temperature of the medium.

Experimentally, the measurement of the real photons is difficult at low- $p_{T}$ region due to large decay photon background and hadron contamination in the detector. In this $p_{T}$ region, $1<p_{T}<$ $5 \mathrm{GeV} / c$, the direct photon yield is reconstructed via virtual photons from dielectron pairs (internal conversion, $\gamma^{*} \rightarrow e^{+} e^{-}$) [2]. Any source of real photons must also emit virtual photons which convert to low-mass $e^{+} e^{-}$pairs. The yield of real and virtual photons is approximately the same in the quasireal photon region $\left(p_{T} \gg m_{e^{+}} e^{-}\right)$. The low-mass distribution of $e^{+} e^{-}$pairs are fitted in mass region $0.1<m_{e^{+} e^{-}}<0.3 \mathrm{GeV} / c^{2}$ to eliminate the $\pi^{0}$ Dalitz decays, and extrapolated to $m_{e^{+} e^{-}} \rightarrow 0$ to obtain the real photon yield.

The PHENIX experiment has measured the photon yield in $\mathrm{Au}+\mathrm{Au}$ at $\sqrt{s_{N N}}=200 \mathrm{GeV}$ in the $p_{T}$ region where the thermal radiation is expected $\left(p_{T}<3 \mathrm{GeV} / c\right)$ and compared it to the spectrum obtained from the $p+p$, see Fig 6 left [2]. The direct photon spectrum in $p+p$ collision was fitted with a modified power-law fit. The minimum bias $\mathrm{Au}+\mathrm{Au}$ collisions show larger photon yield in the low- $p_{T}$ part when compared with the $T_{\mathrm{AA}}$ scaled up $\mathrm{p}+\mathrm{p}$ cross section. The excess yield in the $1-4 \mathrm{GeV} / c$ region in the most central $\mathrm{Au}+\mathrm{Au}$ collisions (0-20\%) is fitted by an exponential function with an inverse slope of $T=221 \pm 19^{\text {stat }} \pm 19^{\text {syst }} \mathrm{MeV}$. If the direct photons in Au+Au collision are of thermal origin, the inverse slope $T$ is related to the initial temperature $\left(T_{\text {init }}\right)$. In hydrodynamical models, the $T_{\text {init }}$ is 1.5 to 3 times $T$ due to space-time evolution [2]. 


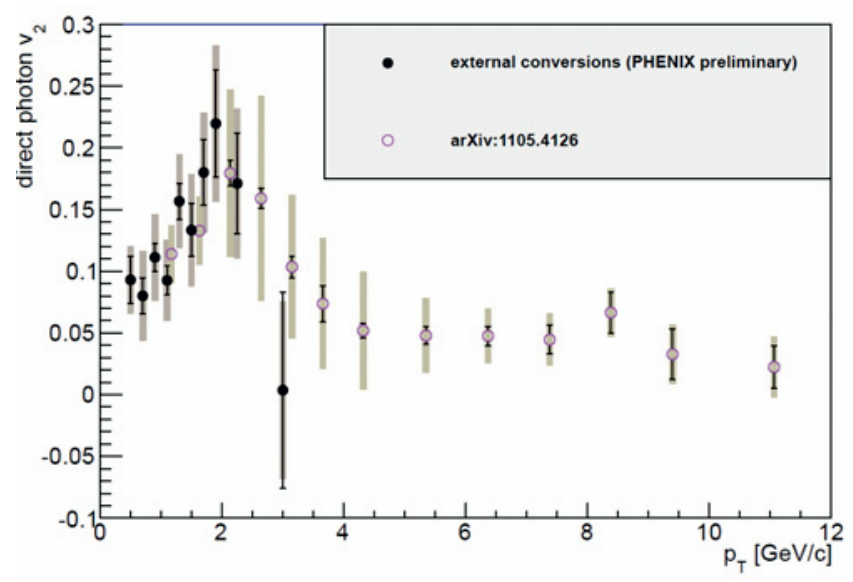

Figure 7. Direct photon azimuthal anisotropy $\left(v_{2}\right)$ in Au+Au collisions at $\sqrt{s_{N N}}=200 \mathrm{GeV}$. The measurement was done with two independent methods, electromagnetic calorimetry with internal conversion of photons and external conversion at $\approx 60 \mathrm{~cm}$ from the interaction point. The error bars represent the statistical uncertainty, while the bands give the systematic uncertainties.

The low- $p_{T}$ region is compared with several hydrodynamical models of thermal photon emissions [2]. The models assume the initial thermalization time around $\tau_{0} \simeq 0.15-0.6 \mathrm{fm} / c$ and the initial temperature ranging $T_{\text {init }} \simeq 300-600 \mathrm{MeV}$. The initial temperature of the medium clearly exceeds the predicted phase transition temperature from hadronic phase to QGP $\left(T_{C} \simeq 170 \mathrm{MeV}\right)$.

Another interesting probe of the medium is the azimuthal anisotropy of the thermal photons. The photon flow depends on the temperature of the medium and the dynamical flow of the medium. The expected azimuthal anisotropy of direct photons from hydrodynamical models is small, as the large fraction of the photons are coming from the QGP phase from early times where the flow of the medium is not formed yet.

The new measurement of the direct photon azimuthal anisotropy, $v_{2}$, has been done in $\mathrm{Au}+\mathrm{Au}$ collisions in $1<p_{T}<13 \mathrm{GeV} / c$ at $\sqrt{s_{N N}}=200 \mathrm{GeV}$. The event plane was determined by the BBC and RXN detectors. The measurement was done with two independent methods: (i) using real photon combined with internal conversion of direct photon measurement [2] and (ii) the conversion of photons $\approx 60 \mathrm{~cm}$ away from the interaction point [2]. The large elliptic flow of the direct photons was observed in heavy ion collisions, where both analysis agree within uncertainties in Fig 7.

The positive direct photon $v_{2}$ which is observed is comparable in the magnitude to the $\pi^{0} v_{2}$ and consistent with early thermalization times and low viscosity of the medium. The magnitude of the direct photon $v_{2}$ is much larger than current theory models predict $[2,2]$. The large thermal photon yield suggests the high initial temperatures of the plasma when the flow of the medium was not developed. On other hand the large photon flow could be formed in later stage of the expansion when the temperature was lower. The large yield and azimuthal anisotropy results simultaneously are not explained by theoretical models $[2,2]$.

\section{Summary}

We studied the $R_{\mathrm{AA}}$ for the opacity and the $v_{n}$ for the fluidity to investigate the presence and properties of the QGP in $\mathrm{Au}+\mathrm{Au}$ collisions at range $\sqrt{s_{N N}}=39-200 \mathrm{GeV}$. The suppression of high- $p_{T}$ 
$\pi^{0}$ decreases towards smaller c.m. energies, but does not disappear. However, there is no significant difference in the charged particle $v_{n}$ coefficients in the studied c.m. energy range. The energy scan at RHIC continues to lower energies also, such as $\sqrt{s_{N N}}=7.7,19$ and $27 \mathrm{GeV}$.

The direct photon production in the hard-scattering region $p_{T}>5 \mathrm{GeV} / c$ in $\mathrm{Au}+\mathrm{Au}$ collisions at $\sqrt{s_{N N}}=200 \mathrm{GeV}$ is described by the scaled $\mathrm{p}+\mathrm{p}$ cross section at the same c.m. energy. The direct photon production in $\mathrm{Au}+\mathrm{Au}$ collision in the thermal region $\left(p_{T}<3 \mathrm{GeV} / c\right)$ shows an excess compared to binary scaled $\mathrm{p}+\mathrm{p}$ collisions. The azimuthal anisotropy of the direct photons was measured in $\mathrm{Au}+\mathrm{Au}$ collisions at $\sqrt{s_{N N}}=200 \mathrm{GeV}$. The large thermal photon yield suggests the photons were produced at early times, in the QGP phase when the temperature was the highest. On other hand the large azimuthal anisotropy of photons is comparable to hadrons that are produced in later time of the fireball evolution.

\section{References}

[1] K. Adcox et al., Nucl.Phys. A757, 184 (2005).

[2] J. Adams et al., Nucl.Phys. A757, 102 (2005).

[3] C. Ratti et al., Nucl.Phys. A855, 253 (2011).

[4] E. V. Shuryak, Phys.Rept. 61, 71 (1980).

[5] M. Strikman and W. Vogelsang, Phys.Rev. D83, 034029 (2011).

[6] S. A. Bass et al., Phys.Rev. C79, 024901 (2009).

[7] U. W. Heinz, arXiv:0901.4355 (2009).

[8] S. A. Voloshin, A. M. Poskanzer, and R. Snellings, (2008).

[9] M. L. Miller, K. Reygers, S. J. Sanders, and P. Steinberg, Ann.Rev.Nucl.Part.Sci. 57, 205 (2007).

[10] K. Adcox et al., Phys.Rev.Lett. 88, 022301 (2002).

[11] C. Adler et al., Phys.Rev.Lett. 89, 202301 (2002).

[12] A. Adare et al., Phys.Rev.Lett. 101, 232301 (2008).

[13] S. Adler et al., Phys.Rev.Lett. 98, 172302 (2007).

[14] A. Adare et al., Phys.Rev.Lett. 101, 162301 (2008).

[15] A. Adare et al., arXiv:1204.1526 (2012).

[16] L. Apanasevich et al., Phys.Rev. D68, 052001 (2003).

[17] T. Sjostrand, S. Mrenna, and P. Z. Skands, Comput.Phys.Commun. 178, 852 (2008).

[18] K. Adcox et al., Phys.Rev.Lett. 89, 212301 (2002).

[19] S. Turbide, C. Gale, E. Frodermann, and U. Heinz, Phys.Rev. C77, 024909 (2008).

[20] S. Afanasiev et al., arXiv:1205.5759 (2012).

[21] A. Adare et al., Phys.Lett. B670, 313 (2009).

[22] D. G. d'Enterria and D. Peressounko, Eur.Phys.J. C46, 451 (2006).

[23] A. Adare et al., Phys.Rev.Lett. 109, 122302 (2012).

[24] R. Petti, J.Phys.Conf.Ser. 316, 012026 (2011).

[25] H. Holopainen, S. Rasanen, and K. J. Eskola, Phys.Rev. C84, 064903 (2011).

[26] H. van Hees, C. Gale, and R. Rapp, Phys.Rev. C84, 054906 (2011). 